\title{
Influence of Periapical Pathosis Upon Postoperative Pain Following Primary Root Canal Treatment in a Single Session Approach
}

\author{
Mostafa I Negm* \\ Lecturer of Endodontics, Faculty of Oral and Dental Medicine, Ahram Canadian \\ University, Egypt \\ *Corresponding Author: Mostafa I Negm, Lecturer of Endodontics, Faculty of Oral \\ and Dental Medicine, Ahram Canadian University, Egypt.
}

DOI: $10.31080 /$ ASDS.2020.05.1021
Received: December 21, 2020

Published: January 18, 2021

(C) All rights are reserved by Mostafa I Negm.

\begin{abstract}
Introduction: This clinical study examined the influence of periapical pathosis of pulpal origin upon postoperative pain following root canal treatment in a single session approach in comparison to those with irreversible pulpal diseases and healthy periodontium. Methods: Thirty patients, between 20-65 years old with thirty molars indicated for primary non-surgical root canal treatment were divided into two groups; group I with irreversible pulpal inflammation and group II with periapical pathosis of pulpal origin. Access cavity preparation performed followed by chemo-mechanical disinfection through hybridization in preparation technique and irrigation protocol. Obturation performed using Resin sealer and cold lateral compaction technique. The primary outcome was postoperative pain which was recorded using a verbal rating scale (VRS) with well-defined categories at the 2-time intervals after root canal treatment: 12 hours and 24 hours. The secondary outcome was analgesics intake. Data was analyzed using the Mann-Whitney U, Fisher's exact, Chi-square tests, and Student's t-test $(\mathrm{P} \leq 0.05)$.

Results: There was no statistically significant difference between median pain scores after 12 (P-value $=0.307$ ) and 24 hours (Pvalue $=0.630$ ) for both groups. There was no statistically significant difference in the intake of analgesics between the two groups (P-value $=1.000)$. There was a statistically significant decrease in pain scores after 12 hours followed by non-statistically significant change in pain scores from 12 to 24 hours in both groups.

Conclusion: Neither postoperative pain nor analgesics intake were influenced by presence of periapical pathosis. However single session root canal treatment resulted in a dramatic decrease in pain intensity irrespective to presence or absence of periapical pathosis.

Keywords: Postoperative Pain; Root Canal Treatment; Single Session; Pulpal Pathosis; Periapical Pathosis
\end{abstract}

\section{Introduction}

The classic study by Kakehashi., et al. in 1965 has verified that pulpal and periapical diseases are microbial in origin [1]. Endodontic infection is a polymicrobial infection mostly obligate anaerobes, predominantly gm -ve that mediates several inflammatory and immunological responses in dental pulp that end mostly by pulp necrosis due to unique features of dental pulp [2]. Unfortunately, there is no natural barrier to prevent dissemination of microorganisms form infected root canals to apical tissues. The elimination of microorganisms emerging from root canals to apical tissues is the function of apical periodontitis [3]. Thus, Apical periodontitis is considered a successful host response to bacteria and bacterial byproducts emerging form infected root canal system as infected pulp canal space acts as a reservoir of infection for periradicular tissues.

The primary objective of endodontic therapy is prevention or treatment of apical periodontitis through maximum root canal disinfection and prevention of reinfection. Many studies showed that complete sterilization of root canal system might be impossible so, the main objective of the preparatory phase of endodontics is to maximally disinfect the root canal system followed by optimum 
three dimensional obturation to resume the early started disinfection protocol via entombing the remaining microorganisms or killing it by a sealer component [4]. Thus, Maximum disinfection of the root canal system is achieved both mechanically by instrumentation as well as chemically through irrigation, medication and optimum three-dimensional obturation. With recent advances in rotary $\mathrm{Ni}$-Ti files heat treatments, kinematics and irrigant agitation as well as obturation techniques; there is an increased tendency towards the single session root canal treatment approach. Many published studies did not demonstrate improvement in the disinfection quality through additional appointments with the advantage of intracanal medicament application and showed that three dimensional obturation of root canal system is the best medication $[5,6]$. The Single session treatment approach has many advantages over other approaches. To name a few; immediate isolation of the root canal system from surrounding periodontium for prevention or treatment of apical periodontitis and immediate fabrication of a post-retained esthetic restoration following traumatic injuries or restorative failure.

Pain is generally known as an unpleasant sensation or emotional experience associated with actual or potential tissue damage. Hence, pain is useful in indicating an ailment that needs to be treated. It is universally agreed that odontogenic pain is best understood as having three organizational elements which are detection, processing, and perception. Detection is the function of peripheral trigeminal pain system via G-protein coupled receptors expressed on the sensory afferent neurons responsible for detecting the intensity, quality, and temporal characteristics of innocuous or noxious stimuli. Processing and perception are the function of the central trigeminal pain system in central brain stem nuclear complex where there is integration and processing of nociceptive input. Processing of the received input can result either in amplification or diminution of peripheral nociceptive input and finally perception [7].

Unfortunately, postoperative pain is a common sequela after endodontic therapy with $3-58 \%$ reported incidence [8]. Several factors might influence the degree of postoperative pain following single session root canal treatment such as psychological condition of patient, gender, preoperative pulpal and periapical status, inten- sity of preoperative pain, change in the ecosystem of root canals and surrounding periodontium, root canal preparation technique, irrigation protocol, method of working length determination and finally the obturation technique $[9,10]$. Flare-up is defined as an acute exacerbation of a pulpal or periapical pathosis represented clinically as severe pain and/or swelling following endodontic therapy. There is almost a universal agreement that teeth with symptomatic and asymptomatic irreversible pulpitis are the best candidates for single session root canal treatment as the periodontium is healthy with a lower risk of postoperative pain or flare-up [11]. Away from acute periapical abscess that requires pus drainage either through root canal or the attachment apparatus, single session root canal treatment for teeth with long standing pulpal disease and secondary periapical pathosis is a matter of controversy. To the best of our knowledge, no published article investigated the influence of periapical pathosis upon postoperative pain following single session root canal treatment in comparison to those with irreversible pulpal diseases and healthy periodontium. The null hypothesis of this clinical study states that periapical pathosis has no influence on the intensity of post-operative pain following a single session root canal treatment.

\section{Methods}

This controlled clinical study was conducted with approval of the Ethics committee of the Faculty of Oral and Dental Medicine at Ahram Canadian University-Egypt. An informed consent form was formulated to include the aim of study, sequence of steps, benefits and risks. Upon consent to participate in the study, all data of the participants were stored securely in locked files in areas with limited access to ensure patients' confidentiality.

\section{Eligibility criteria}

The inclusion criteria were as follows

- Healthy person between the ages of 20 and 65 years old.

- $\quad$ Both males and females were included.

- The offending tooth should be a molar either maxillary or mandibular, which was indicated for primary root canal treatment.

- $\quad$ One molar for every patient. 
The exclusion criteria were the following

- The offending tooth has previous attempts of pulp therapy or root canal treatment.

- $\quad$ The patient represented to the dental office with suppurative periapical pathosis such as acute periapical abscess or cellulitis.

- Any molar that showed pus drainage through root canal in group B upon apical patency concept implementation had been excluded from the study and treated in multiple session approach.

- Patients received systemic antibiotic in the last month.

- Patient received analgesic 12 hours before treatment.

- $\quad$ Offending molar with mobility score $\geq 2$.

- Offending molar with pocket depth $\geq 4 \mathrm{~mm}$.

- Immature molars.

- Nonodontogenic pain.

- Patients with more than one tooth requiring endodontic intervention.

- Intentional root canal treatment in healthy pulp for restorative purpose or aesthetic purpose.

Sample size and grouping

Thirty patients were included in the current study, the power analysis used pain (Verbal Rating Scale) as the primary outcome and analgesics consumption as a secondary outcome. The effect sizes $w 1=(0.812)$ and $w 2=(0.772)$ were calculated based on the results of ElMubarak., et al. [12]. Using alpha ( $\alpha)$ level of (5\%) and Beta $(\beta)$ level of $(20 \%)$ i.e. power $=80 \%$; the minimum estimated sample size was a total of 26 patients. The number will be increased to a total of 30 patients (15 patients per group) to compensate for a dropout rate of $15 \%$. Sample size calculation was performed using G*Power Version 3.1.9.2.

Demographic data including age, sex, address, phone number as well as medical and dental history were recorded in the diagnos- tic charts. The chief complaint was obtained in the patient's own words. Detailed dental history was obtained to reach a tentative diagnosis.

The offending tooth was inspected visually for any caries, cracks or color change in the clinical crown. All non-restorable teeth were excluded from this study. Pocket depths were measured to detect any compromised attachment apparatus and any evidence of root fracture. Palpation and percussion test were also performed to detect any involvement of periapical tissues. Thermometric evaluation of pulpal neural element was performed using both cold (Frijet, ACTEON, Merignac, France) and hot (Dual calamus, DENTSPLY MAILLEFER, Baillagues, Switzerland) sensibility tests. The offending molar and its attachment apparatus were assessed by a periapical digital radiograph (ATECO sensor, Kaso group, England). Based on objective and subjective symptoms as well as clinical and radiographic examination, a definitive clinical diagnosis was reached. The participants of this study were equally divided into two groups according to the presence of periapical pathosis; clinically based on pain localization and radiographically based on the widening of periodontal membrane space or the presence of periapical radiolucency. Group I had healthy periodontium and group II had periapical pathosis. Please refer to figure 1.

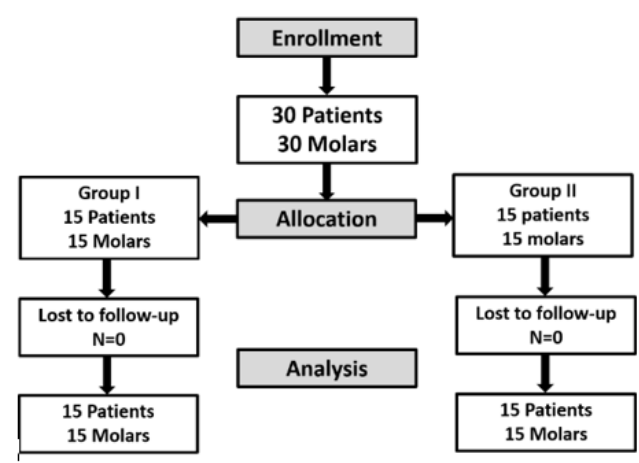

Figure 1: CONSORT 2010 flow diagram of the study. 


\section{Clinical procedures}

All patients were treated by the same clinician in a single session approach irrespective of the group. All molars were anesthetized either through infiltration in case of maxillary molars or inferior alveolar nerve block in case of mandibular molars using Octocaine 2\% with epinephrine 1: 100,000 (Lidocaine $\mathrm{HCl}$, Novocol Pharmaceutical, Ontario, Canada.). A rubber dam was applied, and complete caries removal was followed by opening access using the Endo access bur where restorative diagnosis confirmed the initial clinical diagnosis. Supplemental intraligamental anesthetic technique was applied for patients suffering from any pain during access opening. The mechanical glide path was established using rotary Ni-Ti Proglider file (DENTSPLY MAILLEFER, Baillagues, Switzerland) in a reciprocation maneuver and then early coronal flaring was performed using Gates Glidden drill \#3 in a brushing motion away from the dangerous zone. Supplemental intrapulpal or pressure anesthetic technique was applied for any patient suffering pain during scouting of root canals. Root canals were irrigated using $2.5 \%$ sodium hypochlorite $\mathrm{NaOCl}$ (Clorox; Egyptian Company for household bleach, Egypt) delivered using 30 Gauge safety Steri Irrigation Tip (DiaDent Group International, Burnaby, $\mathrm{BC}$, Canada) $3 \mathrm{~mm}$ below cementoenamel junction to improve the efficiency of electronic apex locator Root ZX II (J. Morita Mfg. Corp, Kyoto, Japan) during working length determination using manual patency stainless-steel K-file \#10. The patency file was advanced just beyond "apex" point on EAL to ensure patency and then drawn back to "apex" point and finally working length was adjusted at anatomic apex. Working length was confirmed radiographically using digital radiography. Root canals were shaped using ProTaper Next rotary Ni-Ti files (DENTSPLY MAILLEFER, Baillagues, Switzerland) in a continuous rotation maneuver. Canals were chemically disinfected using $10 \mathrm{ml} 2.5 \% \mathrm{NaOCl}$, which was delivered $3 \mathrm{~mm}$ coronal to anatomic apex taking into consideration needle is not bound to canal wall. Working length was periodically confirmed electronically during shaping. After complete shaping of root canals, final irrigation with $2.5 \% \mathrm{NaOCl}$ and $17 \%$ EDTA were hydro-dynamically agitated using EndoActivator device (Dentsply Tulsa Dental, Tulsa, OK) with red tips \#25/04 inserted 3mm short of working length for 60 seconds for each irrigant respectively and finally root ca- nals were dried with paper points. Apical patency was confirmed and maintained till obturation using K-file \#10 and an electronic apex locator. Finally, Root canals were obturated using the cold lateral compaction technique and epoxy resin-based sealer (AH plus). Please refer to figure $2,3,4,5$. The remaining amount of tooth structure was evaluated to decide on permanent restoration for each molar. Some molars were restored with an intraradicular metallic post (Unimetric, DENTSPLY MAILLEFER, Baillagues, Switzerland) and coronal dual cure composite restoration (PARKELL, Directa Dental Group, USA) as well as extra coronal zirconia crowns fabrication, while others were restored through zirconia endo crown fabrication. Occlusion was then checked and adjusted in all types of restoration performed. All patients received a prescription of 400 mg ibuprofen every 6 hours in case of severe postoperative pain. All participants were instructed to call for emergency treatment at any time in case of severe postoperative pain not relieved by analgesics.

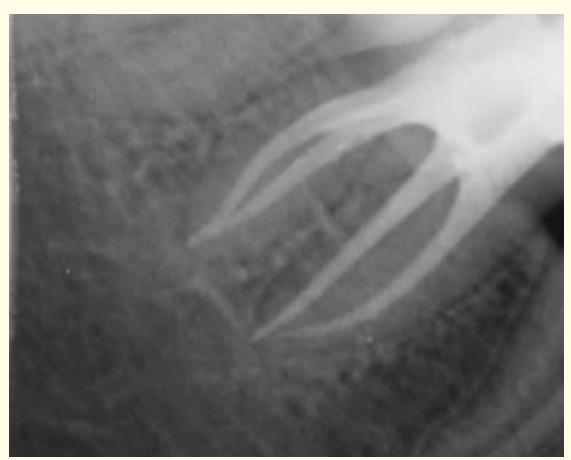

Figure 2: Postoperative radiograph of case no.1 from Gp I.

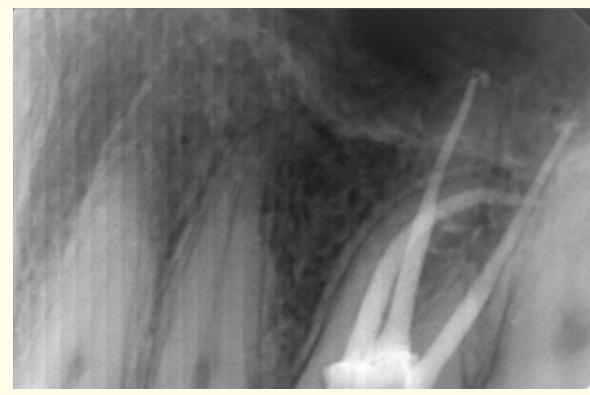

Figure 3: Postoperative radiograph of case no.2 from Gp I. 


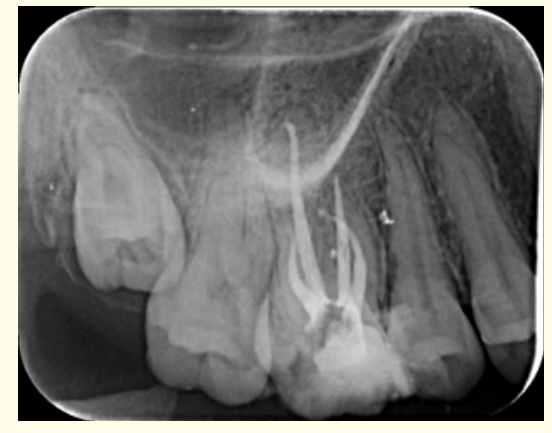

Figure 4: Postoperative radiograph of case no.1 from Gp II.

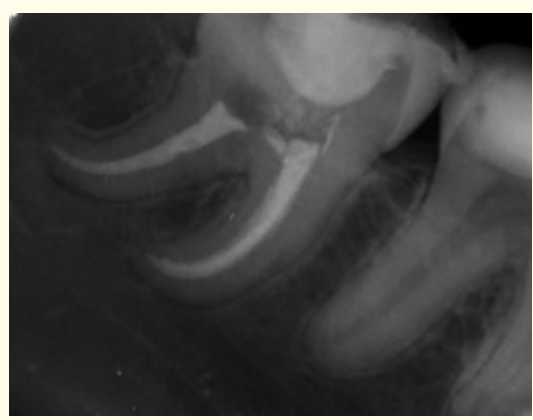

Figure 5: Postoperative radiograph of case no.2 from Gp II.

\section{Patient questionnaire}

All participants calibrated their intensity of preoperative pain in a 4-points numerical verbal rating scale (VRS) questionnaire to be trained on quantifying their postoperative pain at 2-time intervals after root canal treatment; 12 hours and 24 hours. In patients suffering postoperative pain, the frequency of analgesic intake and the time elapsed till complete resolution of symptoms were monitored. Three days after treatment, patients returned to the dental clinic with the filled-in questionnaire and reported their postoperative pain and frequency of analgesic intake. Please refer to results in table 1. The VRS categories were as follows:

- No pain: The treated tooth felt normal.

- Mild pain: Slight discomfort, no treatment needed.

- Moderate pain: Pain relieved by analgesics.

- Severe pain or flare-up: Pain and/or swelling not relieved by analgesics and required an emergency appointment.

\begin{tabular}{|c|l|l|l|l|}
\hline Name & \multicolumn{5}{|l|}{} \\
\hline Age & \multicolumn{5}{|l|}{} \\
\hline Gender & \multicolumn{5}{|l|}{} \\
\hline Arch & \multicolumn{4}{|l|}{} \\
\hline Tooth No. & \multicolumn{4}{|l|}{} \\
\hline Clinical diagnosis & \multicolumn{4}{|l|}{} \\
\hline Group & 1 & 2 & 3 & 4 \\
\hline Pain intensity VRS & & & & \\
\hline Preoperative pain & & & & \\
\hline 12 hours postoperative pain & & & & \\
\hline 24 hours postoperative pain & & & & \\
\hline Analgesic intake & & & \\
\hline
\end{tabular}

Table 1: Questioner for demographic information and assessment of postoperative discomfort as well as analgesic intake.

\section{Statistical analysis}

Qualitative data were presented as frequencies and percentages. Chi-square and Fisher's Exact tests were used for comparisons between the two groups. Numerical data were explored for normality by checking the distribution of data and using tests of normality (Kolmogorov-Smirnov and Shapiro-Wilk tests). Age data showed parametric distribution while pain scores showed nonparametric distribution. Numerical data were presented as mean, standard deviation (SD), median and range values. For parametric data; Student's t-test was used to compare between mean age values in the two groups. For non-parametric data; Mann-Whitney U test was used to compare between the two groups. Friedman's test was used to study the changes within each group. Dunn's test was used for pair-wise comparisons when Friedman's test was found to be significant. The linear regression model was used to determine significant predictors of pain using pain scores as the dependent variable while age, gender, clinical diagnosis, presence of pre-operative pain and intake of analgesics were the independent variables. The significance level was set at $\mathrm{P} \leq 0.05$. Statistical analysis was performed with IBM SPSS Statistics for Windows, Version 23.0. Armonk, NY: IBM Corp.

\section{Results}

No significant difference was found between the participants age $(\mathrm{P}$-value $=0.446)$ and gender $(\mathrm{P}$-value $=0.715)$ as well as offending arch (P-value=0.715) (Table 2). The results showed that 
clinical diagnosis was a statistically significant predictor of preoperative pain while age and gender were not. Patients with asymptomatic irreversible pulpitis, necrotic pulp with asymptomatic apical periodontitis and pulp necrosis showed lower median pain scores than those with necrotic pulp, symptomatic apical periodontitis and those with symptomatic irreversible pulpitis. Please refer to table 3 , figure 6 .

\begin{tabular}{|c|c|c|c|}
\hline & $\begin{array}{l}\text { Group I } \\
(n=15)\end{array}$ & $\begin{array}{l}\text { Group II } \\
(n=15)\end{array}$ & $P$-value \\
\hline \multicolumn{4}{|l|}{ Age (Years) } \\
\hline Mean (SD) & $39.7(12.5)$ & $43.3(13)$ & 0.446 \\
\hline \multicolumn{3}{|l|}{ Gender $[\mathrm{n}(\%)]$} & \multirow{3}{*}{0.715} \\
\hline Female & $8(53.3 \%)$ & $7(46.7 \%)$ & \\
\hline Male & $7(46.7 \%)$ & $8(53.3 \%)$ & \\
\hline \multicolumn{3}{|l|}{$\operatorname{Arch}[\mathrm{n}(\%)]$} & \multirow{3}{*}{1.000} \\
\hline Mandibular & $8(53.3 \%)$ & $8(53.3 \%)$ & \\
\hline Maxillary & 7 (46.7\%) & $7(46.7 \%)$ & \\
\hline
\end{tabular}

Table 2: Descriptive statistics and results of Student's t-test and Chi-square test for comparison between demographic data in the two groups.

\begin{tabular}{|c|c|c|c|}
\hline $\begin{array}{c}\text { Significant } \\
\text { predictors (inde- } \\
\text { pendent variables) }\end{array}$ & $\begin{array}{c}\text { Regression } \\
\text { coefficient } \\
\mathbf{( \beta )}\end{array}$ & $\begin{array}{c}\mathbf{9 5 \%} \text { CI for } \\
\mathbf{( \beta )}\end{array}$ & $\boldsymbol{P}$-value \\
\hline Clinical diagnosis & 0.671 & $0.389-0.944$ & $<0.001^{*}$ \\
\hline
\end{tabular}

Table 3: Linear regression model results showing that clinical diagnosis is a statistically significant predictors of pre-operative pain scores.

No significant difference was found between the two groups regarding the preoperative median pain score $(\mathrm{P}$-value $=0.589$, Effect size $=0.175$ ). All participants could be followed-up for median pain score analysis after 12 and 24 hours. Interestingly, there was also no significant difference between groups in terms of postoperative median pain score neither after 12 hours nor after 24 hours (Pvalue $=0.307$, Effect size $=0.346)$ and $(P$-value $=0.630$, Effect size $=$ 0.114 ), respectively. In regard to the changes with time within each group; there was a statistically significant change in pain scores by time in both group I (P-value $=0.001$, Effect size $=0.452$ ) and group II (P-value $=0.006$, Effect size $=0.345)$. Pair-wise comparisons be-

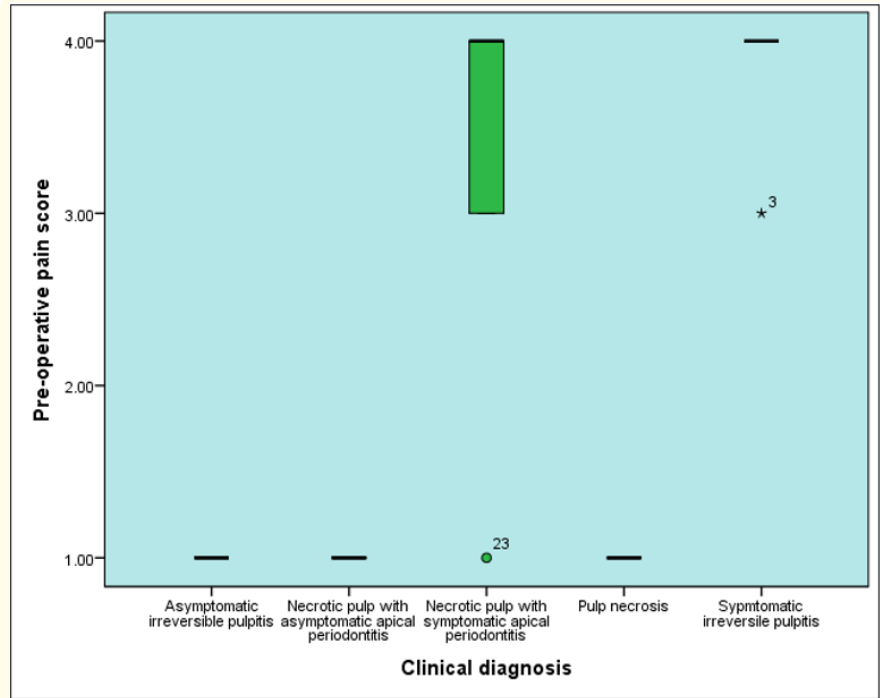

Figure 6: Box plot representing median and range values for pain scores with different clinical diagnosis (Circle and star represent outliers).

tween the time periods revealed that there was a statistically significant decrease in pain scores after 12 hours followed by nonstatistically significant change in pain scores from 12 to 24 hours. Please refer to table 4 and figures 7, 8 .

\begin{tabular}{|c|c|c|c|c|}
\hline Time & $\begin{array}{l}\text { Group I } \\
(n=15)\end{array}$ & $\begin{array}{l}\text { Group II } \\
(n=15)\end{array}$ & $\begin{array}{c}\text { P-value } \\
\text { (between } \\
\text { groups) }\end{array}$ & $\begin{array}{c}\text { Effect } \\
\text { size } \\
\text { (d) }\end{array}$ \\
\hline Pre-operative & & & \multirow[t]{3}{*}{0.589} & \multirow[t]{3}{*}{0.175} \\
\hline Median (Range) & $1(1-4)^{\mathrm{A}}$ & $1(1-4)^{A}$ & & \\
\hline Mean (SD) & $2.33(1.5)$ & $2.07(1.39)$ & & \\
\hline 12 hours & & & \multirow[t]{3}{*}{0.307} & \multirow[t]{3}{*}{0.346} \\
\hline Median (Range) & $1(1-3)^{\text {в }}$ & $2(1-3)^{\mathrm{B}}$ & & \\
\hline Mean (SD) & $1.53(0.74)$ & $1.8(0.77)$ & & \\
\hline 24 hours & & & \multirow[t]{3}{*}{0.630} & \multirow[t]{3}{*}{0.114} \\
\hline Median (Range) & $1(1-2)^{\mathrm{B}}$ & $1(1-2)^{B}$ & & \\
\hline Mean (SD) & $1.13(0.35)$ & $1.2(0.41)$ & & \\
\hline $\begin{array}{l}P \text {-value (Within } \\
\text { group) }\end{array}$ & $0.001^{*}$ & $0.006^{*}$ & & \\
\hline Effect size $(w)$ & 0.452 & 0.345 & & \\
\hline
\end{tabular}

Table 4: Descriptive statistics and results of Mann-Whitney U test for comparison between pain scores in the two groups and Friedman's test for changes by time within each group. 


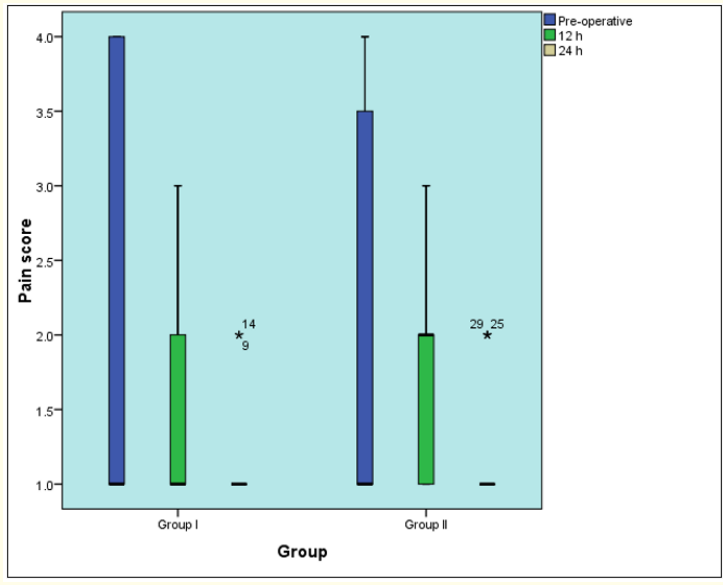

Figure 7: Box plot representing median and range values for pain scores in the two groups (Stars represent outliers).

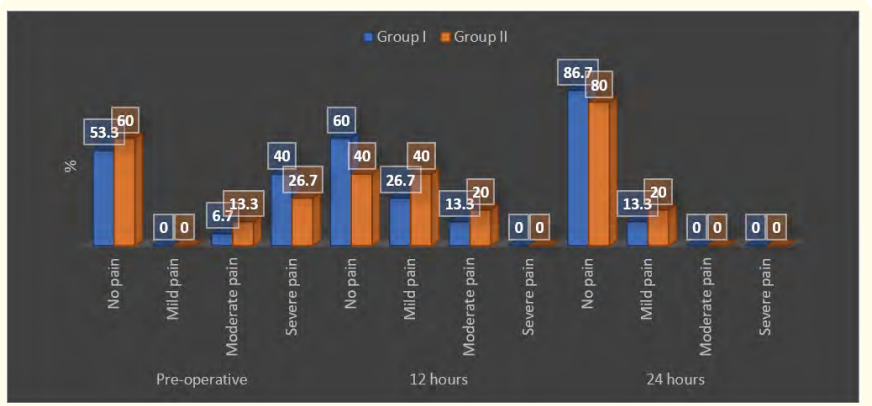

Figure 8: Bar chart representing pain quality in the two groups at different intervals.

A linear regression model was constructed using post-operative pain scores after 12 hours as the dependent variables while age, gender, arch, clinical diagnosis and presence of pre-operative pain were the independent variables. The results showed that presence of pre-operative pain was the only statistically significant predictor of post-operative pain after 12 hours while all other variables were not statistically significant predictors of post-operative pain after 12 hours. The median (Range) values for pain scores were 1 (1 - 1) and 4 (3 - 4) for patients without and with pre-operative pain, respectively. The results showed that none of the independent variables was a statistically significant predictor of post-operative pain after 24 hours. Please refer to table 5 and figure 9.

\begin{tabular}{|c|c|c|c|}
\hline $\begin{array}{c}\text { Significant predictors } \\
\text { (independent } \\
\text { variables) }\end{array}$ & $\begin{array}{c}\text { Regression } \\
\text { coefficient } \\
(\boldsymbol{\beta})\end{array}$ & $\begin{array}{c}\mathbf{9 5 \%} \text { CI for } \\
\mathbf{( \beta )}\end{array}$ & $\boldsymbol{P}$-value \\
\hline $\begin{array}{c}\text { Presence of pre-opera- } \\
\text { tive pain }\end{array}$ & 0.506 & $0.285-$ & $<0.001^{*}$ \\
\hline
\end{tabular}

Table 5: Linear regression model results showing statistically significant predictors of post-operative pain scores after 12 hours.

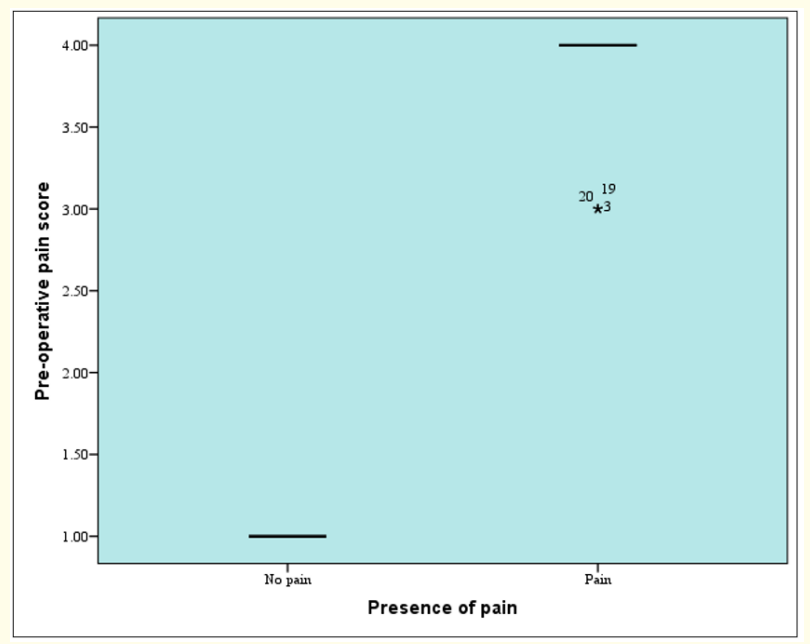

Figure 9: Box plot representing median and range values for pain scores in patients without and with pre-operative pain (Stars represent outliers).

\section{Discussion}

This clinical study evaluated post-operative pain incidence and intensity following primary root canal treatment in a single session approach as well as frequency of analgesic intake. A special emphasis was given in the current study to the effect of periapical pathosis of pulpal origin upon postoperative pain incidence and intensity relative to those with irreversible pulpal pathosis and healthy periodontium. Both preoperative and postoperative pain were calibrated using the numerical 4-points verbal rating scale with scores of no pain, mild pain, moderate pain relieved by analgesic and severe pain and/or flare-up non-responsive to analgesics seeking an emergency appointment [12,13]. Preoperative pain assessment assisted in patient training for calibrating their intensity of pain using verbal rating scale and to investigate influ- 
ence of preoperative pain upon postoperative pain in both groups. Several studies evaluating pain intensity used a visual analogue scale which is a reliable and valid scale [14] but we used verbal rating scale in the current study due to its simplicity as there was a wide range of the patients' educational levels. We evaluated pain intensity at 2-time intervals within 24 hours as several published articles showed maximum postoperative pain during this period and also one article reported that if the patient is free of symptoms $24 \mathrm{~h}$ after obturation, it is unlikely that symptoms will develop during the 60 days after obturation [15]. Molars only were included in the current study as other previous studies showed increased pain intensity in molars following root canal treatment [15].

All treatments were performed by the same clinician of more than 15 years of experience in endodontics to exclude the operator as a variable influencing postoperative pain and to be able to overcome molars' complexities and complete the treatment in a single session approach [16]. During strategy planning for both preparatory and obturation phases, we followed the most recent evidencebased theories minimizing pain sensation either during or following root canal treatment. Every effort was done to minimize both treatment time and iatrogenic errors for more patient convenience and more predictable outcomes. We administrated anesthesia in both groups despite the presence of necrotic pulp in group II to avoid pain during initial scouting of root canal due to remnants of unmyelinated $\mathrm{C}$-fibers which are very resistant to hypoxia and last pulpal element to degenerate [17].

A mechanical glide path was established rather than manual glide path to minimize postoperative pain [18]. As apically extruded debris are one of the major etiologies of postoperative pain, early coronal flaring was performed to minimize apically extruded debris through the creation of wider space for irrigant delivery at an early stage of treatment in addition to straight line access either to primary curvature or physiologic terminus of root canal [19]. Working length determination was postponed till after coronal flaring as several studies reported a decrease in working length in a hundredth of the mm following coronal flaring [20]. Working length was measured electronically and confirmed radiographically for the highest degree of accuracy [21]. The electronic apex locator is the only modality which enables clinicians to apply and maintain apical patency with minimal trauma to the periodontium through slightly advancing the file beyond the apex and then drawing it back to patency point [22]. The Root ZX apex locator was used being the benchmark of all apex locators [23].

The apical patency concept is defined by the American Association of Endodontics as a canal preparation technique where the apical portion of root canal is free of debris by recapitulation of a small file through the apical foramen. Apical patency concept was implemented among the study due to its many advantages such as; the establishment and maintenance of glide path, indicates hidden curvatures of root canals, minimizes apical blockage with minimization of subsequent errors that may be encountered during the re-establishment of the glide path, improves the efficiency of electronic apex locator as a reliable reading cannot be obtained without apical patency concept implementation, relieves pressure in periapical disease with reduced pain intensity, mechanical disruption of microbial biofilm in the apical control zone to provide a predictable level of root canal disinfection and finally enables clinician to obturate root canal three dimensionally up to anatomic apex [24].

M-wire nickel titanium alloy is obtained through thermomechanical processing that resulted in an increased flexibility and improved resistance to cyclic fatigue through increasing the austenitic finish temperature to 50oC $[25,26]$. M-wire Proglider file was used for mechanical glide path establishment in a reciprocation motion $150^{\circ} \mathrm{CW}-30^{\circ} \mathrm{CCW}$ to minimize stresses upon the file and reduce torsional separation to which path files are highly subjected. M-wire Protaper Next files were used in the current study in a continuous rotation motion for shaping of root canals due to its unique geometrical off-centered rectangular cross section that minimizes taper lock [27]. Since the best antidote for file separation is prevention, all files used in the current study were considered a disposable file discarded immediately after use. Irrigation is an integral part of modern endodontic therapy due to nooks and crannies that could not be subjected to mechanical disinfection. Our irrigation protocol throughout the current study was $2.5 \%$ $\mathrm{NaOCl}$ with its unique tissue dissolving action and broad-spectrum 
antimicrobial properties alternatively with $17 \%$ EDTA for smear layer removal [28]. The sonically driven canal irrigation system, EndoActivator was used throughout the current study during root canals disinfection due to its superior ability in cleaning debris, removing smear layer and biofilm dislodgment [29]. Cold lateral compaction obturation technique using Protaper Next gutta percha points and AH plus sealer was used to avoid gross overfilling especially that apical patency concept was implemented throughout the study together with obturation to anatomic apex [30]. We selected anatomic apex as an apical termination point of root canal preparation as well as obturation as Dr. Schilder mentioned in 2006 that he did not encounter a case of endodontic failure due to overfilling but many cases of failure had been encountered due to vertical overextension of underfilled root canals [31].

The basic demographic characteristics [age, gender and arch type] of the two groups showed no statistically significant difference to exclude demographic factor as an influencer of postoperative pain. Similarly, the preoperative median pain scores did not show a statistically significant difference among both groups. Evidently, clinical diagnosis was a significant predictor of preoperative pain where, symptomatic irreversible pulpitis and necrotic pulp with symptomatic apical periodontitis showed the highest median pain scores in both groups.

Interestingly, there was no statistically significant difference between the two groups in terms of postoperative median pain score neither after 12 hours nor after 24 hours confirming the null hypothesis of the current study. Additionally, comparisons between the time periods revealed that there was a statistically significant decrease in pain scores after 12 hours followed by no statistically significant change in pain scores from 12 to 24 hours for both groups ensuring the efficiency of single session root canal treatment for rapid and satisfactory pain relief for both patients and endodontists irrespective to the extent of pulpal diseases. At a 24 hours interval, $13.3 \%$ of patients in group I showed mild postoperative pain whereas, $20 \%$ of cases showed mild pain in group II. None of patients in both groups showed moderate or severe pain at a 24 hours interval and follow-up showed complete pain resolution after 3 days. These results showed that non-surgical root canal treatment is a valid modality for pain relief in a short period of time
[32]. Similarly, there was not a statistically significance difference between the two groups in analgesic intake neither after 12 hours nor after 24 hours.

The current study showed that presence of preoperative pain is the only significant predictor of postoperative pain irrespective of the presence or absence of periapical pathosis [11]. In group I, $86 \%$ of patients with preoperative pain showed postoperative pain with reduced intensity whereas, $100 \%$ of patients with preoperative pain, in group II, showed postoperative pain with reduced intensity. This result can be attributed to central sensitization and/or deafferentation. Central sensitization is the increased excitability of central neurons to noxious stimuli and is a major mechanism of hyperalgesia and allodynia. Central sensitization plays a role in the maintenance of pain after seemingly successful endodontic therapy. Deafferentation refers to the partial or total loss of the afferent nerve supply or sensory input derived from a body region. Numerous studies have shown that endodontic removal of maxillary or mandibular coronal tooth pulps of adult animals induces both morphological changes in trigeminal nerve brain stem nuclear complex which is responsible for postoperative pain [33].

According to the results of the current study, demographic data is not a significant predictor of postoperative pain. On the contrary, other studies have found that preoperative pulpal status is the only predictor of postoperative pain $[17,34]$. The current study showed that gender has no influence on postoperative pain. Other studies reported that gender is one of the prognostic factors influencing postoperative pain [35]. Similarly, the current study showed that age has no influence on postoperative pain. On the contrary, other studies showed that age is among the prognostic factors influencing postoperative pain [11].

Two patients were excluded from the current study as they represented to the dental office with severe localized throbbing pain and their preoperative clinical diagnosis were pulp necrosis with symptomatic apical periodontitis but once the apical patency concept was implemented there was a pus discharge through root canals with dramatic relief of pain; indicating a restorative diagnosis of an early stage of acute periapical abscess with pus embedded inside the bone. Only three asymptomatic patients with slight widening of the periodontal membrane space in group II showed post- 
operative pain with variable intensities after 12 hours [2 patients with moderate pain and one case with severe pain were responsive to the administration of analgesics]. This postoperative pain might be attributed to the alteration in local adaptation syndrome, immunological response with recruitment of neutrophils, change in apical tissue pressure, or treatment-induced change in microbial ecosystem with subsequent release of inflammatory mediators inducing pain [36]. Interestingly, none of the patients from group II with periapical radiolucencies showed any postoperative pain. This finding may be attributed to periapical bone resorption that might tolerate any extruded material asymptomatically in agreement with Torabinejad., et al. [9] and in contrast with Morse., et al. [37] who showed greater flare up tendency in patients with periapical radiolucencies. Only one patient from group II with a clinical diagnosis of pulp necrosis and symptomatic apical periodontitis reported postoperative fever with an elevated body temperature of $38.5 \mathrm{oC}$ and this might be attributed to the release of endotoxins from the killed Gm-ve microorganisms which is usually dominating in symptomatic primary endodontic diseases $[38,39]$.

\section{Conclusions}

Under the limitations of the current study, the following conclusions could be withdrawn:

- Neither postoperative pain nor analgesic consumption was influenced by the presence of periapical pathosis following primary root canal treatment in a single session approach.

- In absence of acute suppurative periapical inflammation, single session root canal treatment is a valid modality for dramatic relief of pain irrespective to the condition of periodontium.

\section{Acknowledgement}

All clinical and laboratory research were funded privately by Mostafa Negm.

The author denies any conflicts of interest related to this clinical study.

\section{Bibliography}

1. Kakehashi S., et al. "The effects of surgical exposures of dental pulps in germ free and conventional laboratory rats". Oral Surgery 20 (1965): 340-349.

2. Sundqvist G. "Bacteriological studies of necrotic dental pulps [deontological dissertations]". Umea, Sweden: University of Umea (1976).

3. Mahmoud Torabinejad., et al. "Inflammatory and immunological aspects of the pathogenesis of human periapical lesions". Journal of Endodontics 11 (1985): 479-488.

4. Sjogren U., et al. "Influence of infection at the time of root filling on the outcome of endodontic treatment of teeth with apical periodontitis". International Endodontic Journal 30 (1997): 297-306.

5. Shuping GB., et al. "Reduction of intracanal bacteria using nickel-titanium rotary instrumentation and various medications". Journal of Endodontics 26 (2000): 751-755.

6. Jorge Paredes-Vieyra., et al. "Success Rate of Single- versus Two-visit Root Canal Treatment of Teeth with Apical Periodontitis: A Randomized Controlled Trial". Journal of Endodontics 38 (2012): 1164-1169.

7. Javier Caviedes-Bucheli., et al. "Neuropeptides in Dental Pulp: The Silent Protagonists". Journal of Endodontics 34 (2008): 773-788.

8. Sathorn C., et al. "The prevalence of postoperative pain and flare-up in single-and multiple-visit endodontic treatment: a systematic review". International Endodontic Journal 41 (2008): 91-99.

9. Mahmoud Torebinejad., et al. "Factors Associated with Endodontic Interappointment Emergencies of Teeth with Necrotic Pulps". Journal of Endodontics 14 (1988): 261-266.

10. Huseyin Sinan., et al. "The Effect of Different Irrigation Agitation Techniques on Postoperative Pain in Mandibular Molar Teeth with Symptomatic Irreversible Pulpitis: A Randomized Clinical Trial". Journal of Endodontics 44 (2018): 1451-1456. 
11. Edward M O’Keefe. "Pain in endodontic therapy: preliminary study". Journal of Endodontics 2 (1976): 315-319.

12. Abdel Hameed H., et al. "Postoperative Pain in Multiple-visit and Single-visit Root Canal Treatment". Journal of Endodontics 36 (2010): 36-39.

13. Yelda Erdem Hepsenoglu., et al. "Postoperative Pain Intensity after Single- versus Two-visit Nonsurgical Endodontic Retreatment: A Randomized Clinical Trial". Journal of Endodontics 44 (2018): 1339-1346.

14. Katsuo Oshima., et al. "Clinical Investigation of Patients Who Develop Neuropathic Tooth Pain After Endodontic Procedures". Journal of Endodontics 35 (2009): 958-961.

15. Nagendrababu V and Gutmann JL. "Factors associated with postobturation pain following single-visit nonsurgical root canal treatment: a systematic review". Quintessence International 48 (2017): 193-208.

16. Daniel Comparin., et al. "Postoperative Pain after Endodontic Retreatment Using Rotary or Reciprocating Instruments: A Randomized Clinical Trial". Journal of Endodontics 43 (2017): 1084-1088.

17. Brannstrom M. "The hydrodynamics of the dental tubule and pulp fluid: its significance in relation to dentinal sensitivity". American Institute of Oral Biology 23 (1966): 219-223.

18. Pasqualini D., et al. "Computed micro-tomographic evaluation of glide path with nickel-titanium rotary PathFile in maxillary first molars curved canals". Journal of Endodontics 38 (2012): 389-393.

19. Alvaro Henrique Borges., et al. "The Influence of Cervical Preflaring on the Amount of Apically Extruded Debris after Root Canal Preparation Using Different Instrumentation Systems". Journal of Endodontics 42 (2016): 465-469.

20. Kyle P Schroeder., et al. "Straight Line Access and Coronal Flaring: Effect on Canal Length". Journal of Endodontics 28 (2002): 474-476.
21. Jorge NR Martins., et al. "Clinical Efficacy of Electronic Apex Locators: Systematic Review". Journal of Endodontics 40 (2014): 759-777.

22. Castellucci A. Endodontics 2 (2002): 42.

23. Plotino G., et al. "Ex vivo accuracy of three electronic apex locators: Root ZX, Elements Diagnostic Unit and Apex Locator and ProPex". International Endodontic Journal 39 (2006): 408-414.

24. Jorge Vera., et al. "Dynamic Movement of Intracanal Gas Bubbles during Cleaning and Shaping Procedures: The Effect of Maintaining Apical Patency on Their Presence in the Middle and Cervical Thirds of Human Root Canals-An In Vivo Study". Journal of Endodontics 38 (2012): 200-203.

25. Alapati SB., et al. "Micro-XRD and temperature-modulated DSC investigation of nickel-titanium rotary endodontic instruments". Dental Material 25 (2009): 1221-1229.

26. Gao Y., et al. "Evaluation of the impact of raw material on the fatigue and mechanical properties of ProFile Vortex rotary instruments". Journal of Endodontics 38 (2012): 398-401.

27. Elio Berutti., et al. "Energy Consumption of ProTaper Next X1 after Glide Path with PathFiles and ProGlider". Journal of Endodontics 40 (2014): 2015-2018.

28. Monica Soares de Albuquerque., et al. "Treatment of an Acute Apical Abscess in a Patient with Autoimmune Hepatitis Taking Alendronate: A Case Report”. Journal of Endodontics 45 (2019): 1550-1555.

29. Neha Verma., et al. "Effect of Different Concentrations of Sodium Hypochlorite on Outcome of Primary Root Canal Treatment: A Randomized Controlled Trial". Journal of Endodontics 45 (2019): 357-363.

30. Indre Graunaite., et al. "Effect of Resin-based and Bioceramic Root Canal Sealers on Postoperative Pain: A Split-mouth Randomized Controlled Trial". Journal of Endodontics 44 (2018): 689-693. 
31. Herbert Schilder. "Filling Root Canals in Three Dimensions". Journal of Endodontics 32 (2006): 281-290.

32. Javier Montero., et al. "Patient-centered Outcomes of Root Canal Treatment: A Cohort Follow-up Study". Journal of Endodontics 41 (2015): 1456-1461.

33. Barry J Sessle. "Recent Developments in Pain Research: Central Mechanisms of Orofacial Pain and Its Control". Journal of Endodontics 12 (1986): 435-443.

34. Hamasha AA and Hatiwsh A. "Quality of life and satisfaction of patients after nonsurgical primary root canal treatment provided by undergraduate students, graduate students and endodontic specialists". International Endodontic Journal 46 (2013): 1131-1139.

35. Watkins CA., et al. "Anticipated and experienced pain associated with endodontic therapy". Journal of the American Dental Association 133 (2002): 45-54.

36. Samuel Seltzer. "Pain in Endodontics". Journal of Endodontics 30 (2004): 501-503.

37. Donald R Morse., et al. "Endodontic Flare-Ups: The Tape". Journal of Endodontics 14 (1988): 106-108.

38. Schein B and Schilder H. "Endotoxin content in endodontically involved teeth". Journal of Endodontics 1 (1975): 19-21.

39. Martinho FC and Gomes BP. "Quantification of endotoxins and cultivable bacteria in root canal infection before and after chemomechanical preparation with $2.5 \%$ sodium hypochlorite". Journal of Endodontics 34 (2008): 268-272.

\section{Assets from publication with us}

- Prompt Acknowledgement after receiving the article

- Thorough Double blinded peer review

- Rapid Publication

- Issue of Publication Certificate

- High visibility of your Published work

Website: www.actascientific.com/

Submit Article: www.actascientific.com/submission.php

Email us: editor@actascientific.com

Contact us: +919182824667 